\title{
Supersymmetric Wilson loops
}

\author{
K. Zarembo* \\ Department of Theoretical Physics \\ Uppsala University \\ Box 803, SE-751 08 Uppsala, Sweden
}

February 1, 2008

\begin{abstract}
I construct $1 / 16,1 / 8$ and $1 / 4$ BPS Wilson loops in $\mathcal{N}=4$ supersymmetric YangMills theory and argue that expectation values of $1 / 4$ BPS loops do not receive quantum corrections. At strong coupling, non-renormalization of supersymmetric Wilson loops implies subtle cancellations in the partition function of the AdS string with special boundary conditions. The cancellations are shown to occur in the semiclassical approximation.
\end{abstract}

\section{Introduction}

The holographic duality of $\mathcal{N}=4$ supersymmetric Yang-Mills theory and type IIB string theory on $A d S_{5} \times S^{5}[1,2,3,4]$ is one of the few examples in which the long suspected equivalence of strings and planar diagrams of the large- $N$ limit [5] can be established on the quantitative level. However, this equivalence is not very explicit in the AdS/CFT correspondence. The string picture is useful in the strong coupling regime of SYM, when all planar diagrams should be taken into account. Resummation of all planar graphs is equivalent to solving the large- $N$ limit, which is impossible in an interacting field theory, such as $\mathcal{N}=4$ SYM.

Though resummation of planar diagrams is a complicated problem in general, simplifications occur in some cases, because supersymmetry leads to cancellations between various contributions. For certain quantities, the supersymmetry constraints are so strong that all quantum corrections cancel. Such quantities do not depend on the coupling and can be computed by summing tree-level diagrams. Non-renormalization theorems of this kind are known to hold for two and three point correlation functions of chiral operators, which preserve $1 / 2$ of $\mathcal{N}=4$ supersymmetry [6]-[17]. The complete sia

*Konstantin.Zarembo@teorfys.uu.se. Also at ITEP, B. Cheremushkinskaya 25, 117259 Moscow, Rus- 
agreement of the supergravity predictions for these correlators with field theory is one of the strongest tests of the AdS/CFT correspondence [18].

Another quantity that is not affected by quantum corrections is an expectation of the straight Wilson line. When properly defined, the expectation value is equal to one, which is confirmed by perturbative computation to the first two orders [19], and also by semiclassical calculations in string theory [20]. A related quantity, the circular Wilson loop, can be obtained from the straight line by a conformal transformation. Though SYM theory is conformally invariant, the expectation value of the circular loop acquires anomalous contributions [21,22] and is a non-trivial function of the coupling constant. This function can be exactly calculated by summing Feynman diagrams that survive supersymmetry cancellations [19]. Various correlation functions of the circular Wilson loop allow to trace how the sum of planar diagrams at weak coupling transforms into the semiclassical string partition function at strong coupling [21, 19, 23, 22].

The cancellation of quantum corrections for the straight Wilson line stems from supersymmetry. The Wilson line operator commutes with eight of the sixteen supercharges [24] and thus corresponds to a BPS state. The circular Wilson loop does not preserve any supersymmetry, but commutes with eight linear combinations of supersymmetry and superconformal generators [25]. Simplifications that occur for the circular loop should be somehow related to its superconformal invariance.

I will construct Wilson loops that preserve $1 / 16,1 / 8$ or $1 / 4$ of the supersymmetry and study their expectation values in perturbation theory and at strong coupling using AdS string theory. Comparing the results of these calculations, I conjecture that the expectation values of $1 / 4$ BPS Wilson loops are not renormalized. But less supersymmetric Wilson loops do receive quantum corrections.

\section{Supersymmetry transformations}

The field content of $\mathcal{N}=4 \mathrm{SYM}$ theory consists of gauge fields $A_{\mu}$, six scalars $\Phi_{i}$ $(i=4 \ldots 9)$ and four Majorana fermions $\Psi^{A}$, all in the adjoint representation of $S U(N)$. It is convenient to put fermions into a single Majorana-Weyl spinor of $\operatorname{Spin}(9,1)$. The Euclidean action then takes the following form:

$$
S=\frac{1}{g^{2}} \int d^{4} x \operatorname{tr}\left\{\frac{1}{2} F_{\mu \nu}^{2}+\left(D_{\mu} \Phi_{i}\right)^{2}-\frac{1}{2}\left[\Phi_{i}, \Phi_{j}\right]^{2}+\bar{\Psi} \Gamma^{\mu} D_{\mu} \Psi+i \bar{\Psi} \Gamma^{i}\left[\Phi_{i}, \Psi\right]\right\},
$$

where $\Gamma^{M}=\left(\Gamma^{\mu}, \Gamma^{i}\right)$ are ten-dimensional Dirac matrices. The supersymmetry transformations of the bosonic field are

$$
\begin{aligned}
\delta_{\epsilon} A_{\mu} & =\bar{\Psi} \Gamma^{\mu} \epsilon, \\
\delta_{\epsilon} \Phi_{i} & =\bar{\Psi} \Gamma^{i} \epsilon,
\end{aligned}
$$

where the parameter of transformation $\epsilon$ is a ten-dimensional Majorana-Weyl spinor.

The Wilson loop operator which is dual to the string in Anti-de-Sitter space [26] is a hybrid of the usual non-Abelian phase factor and the scalar loop of [27]:

$$
W(C, \theta)=\frac{1}{N} \operatorname{tr} \mathrm{P} \exp \int d s\left(i A_{\mu}(x) \dot{x}^{\mu}+\Phi_{i}(x) \theta^{i}|\dot{x}|\right) .
$$


Here, $x^{\mu}(s)$ parameterizes the contour $C$ in $\mathbb{R}^{4}$ and $\theta^{i}$ is a unit six-vector: $\theta^{i} \theta^{i}=1$. This vector can depend on $s$, though, in most of the papers on Wilson loops, $\theta^{i}$ was assumed to be constant.

The supersymmetry variation of the Wilson loop is

$$
\delta_{\epsilon} W(C, \theta)=\frac{1}{N} \operatorname{tr} \mathrm{P} \int d s \bar{\Psi}\left(i \Gamma^{\mu} \dot{x}^{\mu}+\Gamma^{i} \theta^{i}|\dot{x}|\right) \epsilon \exp \int d s^{\prime}\left(i A_{\mu} \dot{x}^{\mu}+\Phi_{i} \theta^{i}|\dot{x}|\right) .
$$

Some part of the supersymmetry* will be preserved if

$$
\left(i \Gamma^{\mu} \dot{x}^{\mu}+\Gamma^{i} \theta^{i}|\dot{x}|\right) \epsilon=0 .
$$

Since the linear combination of Dirac matrices $i \Gamma^{\mu} \dot{x}^{\mu}+\Gamma^{i} \theta^{i}|\dot{x}|$ squares to zero, equation (2.5) has eight independent solutions for any given $s$. In general, these solutions will depend on $s$, so an arbitrary Wilson loop is only locally supersymmetric. Local supersymmetry is not a symmetry of the action, however. The requirement that $\epsilon$ is $s$-independent is a constraint on $x^{\mu}(s)$ and $\theta^{i}(s)$. The number of linearly independent $\epsilon$ 's that satisfy eq. (2.5) determines the number of conserved supercharges.

It is easy to convince oneself that eq. (2.5) has no solutions for constant $\theta^{i}$, unless $C$ is a straight line. Indeed, choosing parameterization of the contour $C$ such that $|\dot{x}|=1$ and differentiating (2.5) in $s$, we get:

$$
i \Gamma^{\mu} \ddot{x}^{\mu} \epsilon=0,
$$

which implies that $\ddot{x}$ is identically zero.

For a general loop with varying $\theta^{i}$ and a curved contour $C,(2.5)$ constitutes an infinite set of algebraic equations for sixteen unknown quantities. In spite of the huge redundancy of these equations, they have non-trivial solutions for certain $x^{\mu}$ and $\theta^{i}$. The complete classification of supersymmetric Wilson loops is beyond the scope of the present paper. Instead, I will study a simple ansatz, for which (2.5) reduces to a finite number of equations. The ansatz amounts in requiring that the position of the loop in $S^{5}$ follows the tangent vector $\dot{x}^{\mu}$ of the space-time contour $C$. The map from $S^{3}$ to $S^{5}$ is defined by an immersion of $\mathbb{R}^{4}$ in $\mathbb{R}^{6}$ as a hyperplane:

$$
x^{\mu} \longmapsto x^{\mu} M_{\mu}^{i}
$$

where the rectangular matrix $M_{\mu}^{i}$ can be regarded as a projection operator:

$$
M_{\mu}^{i} M_{\nu}^{i}=\delta_{\mu \nu}
$$

A particular form of $M_{\mu}^{i}$ is not important because of $S O(4) \times S O(6)$ global symmetry of $\mathcal{N}=4$ SYM. Then,

$$
\theta^{i}=M_{\mu}^{i} \frac{\dot{x}^{\mu}}{|\dot{x}|}
$$

maps a tangent vector of the contour $C$ to a point on $S^{5}$. With this choice of $\theta^{i}$, the Wilson loop operator becomes

$$
W_{s}(C)=\frac{1}{N} \operatorname{tr} \mathrm{P} \exp \oint_{C} d x^{\mu}\left(i A_{\mu}+M_{\mu}^{i} \Phi_{i}\right)
$$

\footnotetext{
*It would be also interesting to consider more general conditions for superconformal invariance of a Wilson loop operator (see [25] for the discussion of the circular loop).
} 
This is my ansatz for the supersymmetric Wilson loop. The supersymmetry variation of this operator vanishes if

$$
i \dot{x}^{\mu}\left(\Gamma^{\mu}-i M_{\mu}^{i} \Gamma^{i}\right) \epsilon=0 .
$$

All $s$-dependence factors out, and one is left with four algebraic equations:

$$
\left(\Gamma^{\mu}-i M_{\mu}^{i} \Gamma^{i}\right) \epsilon=0 .
$$

These equations can be easily solved by choosing a particular basis in the spinor representation of $\operatorname{Spin}(10)^{\dagger}$. Let us define four pairs of creation and annihilation operators:

$$
\begin{aligned}
a^{\mu} & =\frac{1}{2}\left(\Gamma^{\mu}-i M_{\mu}^{i} \Gamma^{i}\right), \\
a_{\mu}^{\dagger} & =\frac{1}{2}\left(\Gamma^{\mu}+i M_{\mu}^{i} \Gamma^{i}\right) .
\end{aligned}
$$

The fifth pair of operators is constructed using two six-vectors orthogonal to $M_{\mu}^{i}$ :

$$
\begin{gathered}
M_{\mu}^{i} v_{1,2}^{i}=0, \quad v_{1,2}^{2}=1, \\
a^{4}=\frac{1}{2}\left(v_{1}^{i} \Gamma^{i}-i v_{2}^{i} \Gamma^{i}\right), \\
a_{4}^{\dagger}=\frac{1}{2}\left(v_{1}^{i} \Gamma^{i}+i v_{2}^{i} \Gamma^{i}\right) .
\end{gathered}
$$

The matrices $a^{M}, a_{M}^{\dagger}$ satisfy anti-commutation relations

$$
\left\{a^{M}, a_{N}^{\dagger}\right\}=\delta_{N}^{M},
$$

and their Fock space can be identified with the spinor representation of $\operatorname{Spin}(10)$. The chirality projection leaves states with even (or odd, depending on the sign of chirality projection) number of creation operators acting on the Fock vacuum.

The equations (2.11) in this representation are

$$
a^{\mu}|\epsilon\rangle=0, \quad \mu=0 \ldots 3 .
$$

For the spinor to be annihilated by $a^{0} \ldots a^{3}$, the levels associated with these oscillators must be filled. There are two such states:

$$
\begin{aligned}
& \left|\epsilon_{+}\right\rangle=a_{0}^{\dagger} \ldots a_{3}^{\dagger}|0\rangle, \\
& \left|\epsilon_{-}\right\rangle=a_{0}^{\dagger} \ldots a_{3}^{\dagger} a_{4}^{\dagger}|0\rangle .
\end{aligned}
$$

They have opposite chirality. Hence, there is only one Weyl spinor that satisfies eqs. (2.11) and, consequently, the Wilson loop operator commutes with one of the sixteen supercharges. So, a Wilson loop of the form (2.9) preserves supersymmetry and generically is $1 / 16$ BPS.

\footnotetext{
${ }^{\dagger}$ Strictly speaking, one should deal with $\operatorname{Spin}(9,1)$ spinors, because of the Majorana condition on $\epsilon$. However, the signature of the metric will not be important for the discussion below, and I will assume that the Dirac matrices anti-commute on $2 \delta^{M N}$ from now on, to simplify the notations.
} 
Table 1: The amount of supersymmetry for Wilson loops of various dimensions.

\begin{tabular}{|c|c|}
\hline Dimensionality of the loop & Amount of supersymmetry \\
\hline 4D & $1 / 16$ \\
3D & $1 / 8$ \\
2D & $1 / 4$ \\
1D & $1 / 2$ \\
\hline
\end{tabular}

The supersymmetry is enhanced if the contour $C$ has a special shape. Consider, for instance, a spatial Wilson loop which lies in a three-dimensional time slice $x^{0}=0 . \dot{x}^{0}$ is identically equal to zero in this case, so only three of four constraints in (2.11), those with $\mu=1 \ldots 3$, should be imposed to satisfy (2.10). Then $|\epsilon\rangle$ must be annihilated only by three oscillators. In addition to (2.17), there are two extra solutions that satisfy these constraints:

$$
\begin{aligned}
\left|\epsilon_{+}^{(1)}\right\rangle & =a_{0}^{\dagger} \ldots a_{3}^{\dagger}|0\rangle, \\
\left|\epsilon_{-}^{(1)}\right\rangle & =a_{0}^{\dagger} \ldots a_{3}^{\dagger} a_{4}^{\dagger}|0\rangle, \\
\left|\epsilon_{+}^{(2)}\right\rangle & =a_{1}^{\dagger} \ldots a_{3}^{\dagger}|0\rangle, \\
\left|\epsilon_{-}^{(2)}\right\rangle & =a_{1}^{\dagger} \ldots a_{3}^{\dagger} a_{4}^{\dagger}|0\rangle .
\end{aligned}
$$

Two of these spinors are chiral, so a three-dimensional loop commutes with 2 supercharges and preserves $1 / 8$ of the supersymmetry. If the contour $C$ lies in a twodimensional plane, the number of supersymmetries again doubles, and the planar Wilson loop preserves $1 / 4$ of the supersymmetry. A one-dimensional supersymmetric Wilson loop is a familiar Wilson line with constant $\theta^{i}$, which is $1 / 2$ BPS. The amount of supersymmetry as a function of dimensionality of the loop is summarized in table 1 .

\section{Supersymmetric Wilson loops in perturbation theory}

One may anticipate a lot of cancellations between quantum corrections for the supersymmetric Wilson loops. I will calculate the expectation value

$$
\left\langle W_{s}(C)\right\rangle=\left\langle\frac{1}{N} \operatorname{tr} \mathrm{P} \exp \oint_{C} d x^{\mu}\left(i A_{\mu}+M_{\mu}^{i} \Phi^{i}\right)\right\rangle
$$

to the two first orders in perturbation theory and to the leading order in the large- $N$ expansion and will demonstrate that all corrections mutually cancel.

There are nine diagrams (fig. 1) that contribute at $O\left(\left(g^{2} N\right)^{2}\right)$. Each of the individual graphs in fig. 1 contains UV divergences. The divergences cancel in the sum, but intermediate calculations require a regularization. An explicit regularization prescription will not be important for the calculations below, as soon as the regularization preserves supersymmetry. One can always keep in mind the dimensional reduction 


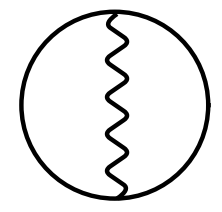

$a$
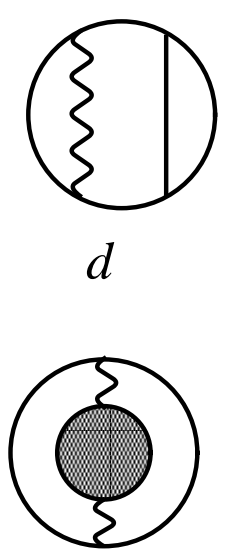

$g$

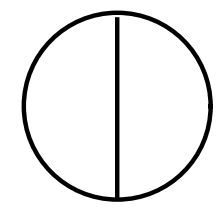

$b$
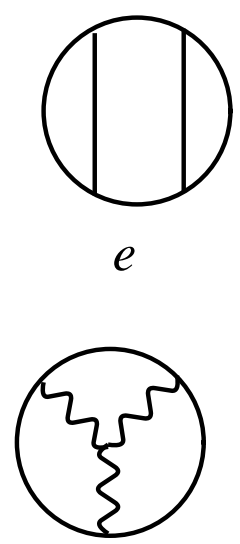

$h$
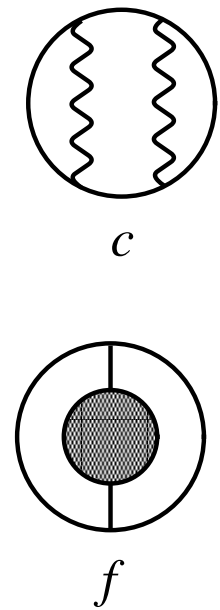

c

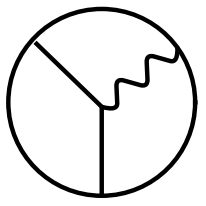

$i$

Figure 1: Feynman diagrams of leading and next-to-leading orders.

which works to this order in perturbation theory $[19,28,29]$. I will not even need an explicit form of propagators. The only necessary ingredient is the equality of gauge boson and scalar propagators in the Feynman gauge, which is a consequence of supersymmetry:

$$
\begin{aligned}
& \left\langle A_{\mu}^{a b}(x) A_{\nu}^{c d}(y)\right\rangle_{0}=g^{2}\left(\delta^{a d} \delta^{b c}-\frac{1}{N} \delta^{a b} \delta^{c d}\right) \delta_{\mu \nu} D(x-y), \\
& \left\langle\Phi_{i}^{a b}(x) \Phi_{i}^{c d}(y)\right\rangle_{0}=g^{2}\left(\delta^{a d} \delta^{b c}-\frac{1}{N} \delta^{a b} \delta^{c d}\right) \delta_{i j} D(x-y),
\end{aligned}
$$

where $a, b, c, d$ are $S U(N)$ indices.

The diagrams of the leading order, (a) and (b), mutually cancel, because

$$
\left\langle\left(i A_{\mu}(x)+M_{\mu}^{i} \Phi_{i}(x)\right)\left(i A_{\nu}(y)+M_{\nu}^{j} \Phi_{j}(y)\right)\right\rangle_{0} \propto\left(i^{2} \delta_{\mu \nu}+M_{\mu}^{i} M_{\nu}^{i}\right) D(x-y)=0,
$$

owing to the identity (2.7). All diagrams without internal vertices cancel for the same reason, in particular, the diagrams (c), (d) and (e). As was shown in [19], one-loop corrected scalar and vector propagators are still equal, up to total derivatives, so the diagrams (f) and (g) also sum up to zero. 
The remaining graphs (h) and (i) are computed as follows:

$$
\begin{aligned}
(h)+(i)= & \frac{2}{g^{2} N} \int d^{4} x \oint d x_{1}^{\mu} d x_{2}^{\nu} d x_{3}^{\lambda} \theta_{c}\left(x_{1}, x_{2}, x_{3}\right) \\
& \times\left(\frac{1}{3}\left\langle\operatorname{tr} A_{\mu}\left(x_{1}\right) A_{\nu}\left(x_{2}\right) A_{\lambda}\left(x_{3}\right) \operatorname{tr} \partial_{\rho} A_{\sigma}(x)\left[A_{\rho}(x), A_{\sigma}(x)\right]\right\rangle\right. \\
& \left.-\left\langle M_{\mu}^{i} M_{\nu}^{j} \operatorname{tr} \Phi_{i}\left(x_{1}\right) \Phi_{j}\left(x_{2}\right) A_{\lambda}\left(x_{3}\right) \operatorname{tr} \partial_{\rho} \Phi_{k}(x)\left[A_{\rho}(x), \Phi_{k}(x)\right]\right\rangle_{0}\right) \\
= & 2\left(g^{2} N\right)^{2} \int d^{4} x \oint d x_{1}^{\mu} d x_{2}^{\nu} d x_{3}^{\lambda} \theta_{c}\left(x_{1}, x_{2}, x_{3}\right) \delta_{\mu \nu} D\left(x-x_{3}\right) \\
& \times\left(D\left(x-x_{1}\right) \partial_{\lambda} D\left(x-x_{2}\right)-\partial_{\lambda} D\left(x-x_{1}\right) D\left(x-x_{2}\right)\right. \\
& \left.-D\left(x-x_{1}\right) \partial_{\lambda} D\left(x-x_{2}\right)+\partial_{\lambda} D\left(x-x_{1}\right) D\left(x-x_{2}\right)\right)=0 .
\end{aligned}
$$

Here, $\theta_{c}\left(x_{1}, x_{2}, x_{3}\right)$ is equal to one, if points $x_{1}, x_{2}$ and $x_{3}$ are cyclically ordered along the contour $C$, and is equal to zero otherwise.

All corrections of orders $g^{2} N$ and $\left(g^{2} N\right)^{2}$ cancel. That may indicate that expectation values of supersymmetric Wilson loops obey non-renormalization theorems. If so, AdS/CFT calculations at strong coupling must also give $\left\langle W_{s}(C)\right\rangle=1$.

\section{Supersymmetric Wilson loops in string the- ory}

According to the AdS/CFT conjecture, Wilson loops couple directly to strings that propagate in $A d S_{5} \times S^{5}$. The expectation value of the Wilson loop is the string partition function $[26,30]$ :

$$
\begin{aligned}
\langle W(C, \theta)\rangle= & \int_{\text {reg }} D X^{M} D \vartheta^{\alpha} D h_{a b} \exp \left(-\frac{\sqrt{g^{2} N}}{4 \pi} \int_{D} d^{2} \sigma \sqrt{h} h^{a b} G_{M N} \partial_{a} X^{M} \partial_{b} X^{N}\right. \\
& + \text { fermions }),
\end{aligned}
$$

The sigma-model metric is defined by the line element:

$$
d \mathfrak{s}^{2}=\frac{1}{Z^{2}}\left(d X_{\mu}^{2}+d Z^{2}\right)+d \Omega_{S^{5}}^{2}
$$

The fermionic part of the action is known [31]-[36], [20], but will not be used here. The string world sheet extends to the boundary of AdS space, where it terminates on the contour $C$. In other words, the sigma-model path integral is supplemented by the boundary conditions:

$$
\left.X^{\mu}\right|_{\partial D}=x^{\mu}(s),\left.\quad Z\right|_{\partial D}=\varepsilon,\left.\quad \Theta^{i}\right|_{\partial D}=\theta^{i}(s) .
$$

$\Theta^{i}$ parameterizes a position of the world sheet in $S^{5}$. The regularization parameter $\varepsilon$ cuts off divergences which arise because of the $1 / z^{2}$ singularity of the metric at the 
boundary. At the end, $\varepsilon$ should be sent to zero. If the string partition function is appropriately defined, the divergences appear only in the intermediate calculations and eventually cancel. The correct definition of the partition function involves the Legendre transform in $Z$ [24]. Explicit implementation of the Legendre transform is somewhat cumbersome, but, fortunately, in the semiclassical approximation, the Legendre transform amounts in dropping $1 / \varepsilon$ divergences whenever they appear.

It is not known how to solve the $A d S_{5} \times S^{5}$ sigma model exactly. The only simplification occurs at large 't Hooft coupling, when the sigma model becomes weakly coupled, and the partition functions can be computed in the saddle-point approximation. Minima of the string action correspond to minimal surfaces in $A d S_{5} \times S^{5}$, whose boundary is the contour $C$. The action at the saddle point is the area of the minimal surface:

$$
A(C)=\int d^{2} \sigma \sqrt{\operatorname{det}_{a b} G_{M N} \partial_{a} X_{\mathrm{cl}}^{M} \partial_{b} X_{\mathrm{cl}}^{N}},
$$

which should be regularized by subtraction of the boundary divergence. The $\alpha^{\prime}$ expansion of the sigma model, obtained by expanding around the classical solution and integrating out fluctuations, yields $1 / \sqrt{g^{2} N}$ expansion of the Wilson loop expectation value. There are additional complications if the classical solution depends on parameters (has moduli). The parameters give rise to zero mode fluctuations which should be separated by introducing collective coordinates for the moduli of the classical solution. Each moduli integration is accompanied by a factor of $\alpha^{1 / 2}$, which, in the present case, should be identified with $\left(g^{2} N\right)^{1 / 4}$. The gauge fixing of the world sheet diffeomorphism / Weyl invariance also produces a non-trivial factor, because the usual conformal gauge leaves residual three-parametric gauge freedom in the disk partition function [37]. The residual gauge symmetries give a factor of $\left(g^{2} N\right)^{-1 / 4}$ each [21]. Consequently, the semiclassical partition function for the Wilson loop vev has the following general form:

$$
\langle W(C, \theta)\rangle=\text { const }\left(g^{2} N\right)^{\left(N_{z . m .}-3\right) / 4} \exp \left(-\frac{\sqrt{g^{2} N}}{2 \pi} A(C)\right)+1 / \sqrt{g^{2} N} \text { correction, }
$$

where $N_{z . m}$. is the number of zero modes, or the number of moduli in the classical solution $X_{\mathrm{cl}}^{M}$. An overall constant comes from the integration over non-zero-mode fluctuations.

Potential non-renormalization of supersymmetric Wilson loops implies that corresponding minimal surfaces have zero area. For loops with constant $\theta_{i}$, which have been most extensively studied so far, the minimal surface sits at one point in $S^{5}$ and extends only in $A d S_{5}$. It can be shown that the regularized area of a minimal surface in $A d S_{5}$ is strictly negative because of the subtraction of the boundary divergence. The area in $S^{5}$ is positive, which may well cancel the $A d S_{5}$ contribution. So, nullification of the classical string action is not very surprising, and I will show that it indeed happens for the simplest contours. The cancellation of the zero mode factor is a much more unexpected fact. For the Wilson loop vev to be one, the minimal surface must be degenerate and must depend exactly on three parameters. A minimal surface with a given boundary in $A d S_{5}$ is usually unique. Several degenerate minima of the string action can coexist in some special cases, but this degeneracy is never parametric. This 
leads to a characteristic $\left(g^{2} N\right)^{-3 / 4}$ pre-factor in the Wilson loop expectation value [21] which is confirmed by exact field-theory calculations [19]. It turns out that a non-trivial dependence on $S^{5}$ coordinates qualitatively changes the situation. Minimal surfaces which are associated with supersymmetric Wilson loops are always parametrically degenerate and have moduli. The number of moduli turns out to depend on the amount of supersymmetry preserved by the Wilson loop. Minimal surfaces with constant position in $S^{5}$ can be regarded as a degenerate case, in which the moduli space shrinks to zero size.

The simplest supersymmetric Wilson loop consists of two anti-parallel lines with points at infinity identified, which can be thought of as a limit of an infinitely long rectangular contour. Supersymmetry requires that $\theta_{i}$ follows the tangent vector of the contour $C$. Since the tangent vector rotates through $\pi$ at infinity, each of the two lines should be put at diametrically opposite points on $S^{5}$. This is a particular case of configuration considered in [26]. It was observed there that the potential between anti-parallel lines vanishes when their angular separation on $S^{5}$ reaches $\pi$. This is exactly where the Wilson loop becomes supersymmetric.

I will consider in detail another simple example, the circular supersymmetric Wilson loop. Due to the scale invariance of $\mathcal{N}=4 \mathrm{SYM}$, the radius of the circle can be put to one, and it can be parameterized as

$$
x^{\mu}(s)=(\cos s, \sin s, 0,0) .
$$

The loop in $S^{5}$ must be an equatorial circle to satisfy the supersymmetry constraints. Choosing the angular parameterization of $S^{5}$, when the metric is

$$
d \Omega_{S^{5}}^{2}=d \psi^{2}+\cos ^{2} \psi d \varphi^{2}+\sin ^{2} \psi d \Omega_{S^{3}}^{2}, \quad 0 \leq \psi<\pi, \quad 0 \leq \varphi<2 \pi,
$$

we have the following boundary conditions for the $S^{5}$ coordinates

$$
\varphi(s)=s, \quad \psi(s)=0, \quad \mathbf{n}=\text { const }
$$

where $\mathbf{n}$ is a unit four-vector that corresponds to a point on $S^{3}$. The minimal surface can be parameterized by the angle $s$ and the AdS scale $z$. Imposing the symmetry constraints, we can look for a solution of the following form:

$$
X^{\mu}=(R \cos s, R \sin s, 0,0), R \equiv R(z), \quad Z=z, \quad \Phi=s, \quad \Psi \equiv \Psi(z) .
$$

The area of this surface is

$$
A=2 \pi \int d z \sqrt{\left(\frac{R^{2}}{z^{2}}+\cos ^{2} \Psi\right)\left(\Psi^{\prime 2}+\frac{R^{\prime 2}+1}{z^{2}}\right)} .
$$

Variational equations following from minimization of $A$ are quite complicated, but the solution is rather simple:

$$
R=\sqrt{1-z^{2}}, \quad \sin \Psi=z .
$$

Checking that this solves equations of motion for the Nambu-Goto action is a straightforward but lengthy exercise. An easier way to see that this corresponds to a minimal surface is outlined below. 
A metric induced on the minimal surface is

$$
d \mathfrak{s}^{2}=\left(\frac{1}{z^{2}}+1\right)\left[\frac{d z^{2}}{1-z^{2}}+\left(1-z^{2}\right) d s^{2}\right]
$$

and the minimal area is readily computed

$$
A(\text { circle })=\int_{0}^{2 \pi} d s \int_{\varepsilon}^{1} d z\left(\frac{1}{z^{2}}+1\right)=\frac{2 \pi}{\varepsilon} .
$$

The regularized area, left after subtraction of the divergence, is zero, which is consistent with non-renormalization of the Wilson loop expectation value: $\left\langle W_{s}(\right.$ circle $\left.)\right\rangle=1$.

The above solution can be easily obtained if we start with the action in the Polyakov form, as in (4.1), and fix the conformal gauge: $h_{a b}=\delta_{a b}$. The equations for the minimal surface then follow from the action

$$
A=\pi \int d \tau\left[\frac{R^{\prime 2}+R^{2}+Z^{\prime 2}}{Z^{2}}+\Psi^{\prime 2}+\cos ^{2} \Psi\right]
$$

where now $R, Z$ and $\Psi$ are functions of $\tau$. The equations should be supplemented by Virasoro constraints, which require the induced metric to be unit matrix up to a conformal factor:

$$
G_{M N} \partial_{a} X^{M} \partial_{b} X^{N}=\mathrm{e}^{\phi} \delta_{a b}
$$

Both the equations of motion and the Virasoro constraints can be solved separately for the $A d S_{5}$ and the $S^{5}$ coordinates of the string world sheet. Hence, the minimal surface in the conformal gauge is a direct sum of two surfaces in $A d S_{5}$ and in $S^{5}$. Minimal surface in $A d S_{5}$ that has a circle as its boundary is known [24, 38]. Transforming the solution of $[24,38]$ to the conformal gauge we get:

$$
R=\frac{1}{\cosh \tau}, \quad Z=\tanh \tau
$$

The $A d S_{5}$ part of the induced metric is

$$
d \mathfrak{s}_{A d S_{5}, \text { induced }}^{2}=\frac{1}{\sinh ^{2} \tau}\left(d \tau^{2}+d s^{2}\right) .
$$

Solving the equation of motion for $\Psi$ with boundary conditions (4.8), we find:

$$
\sin \Psi=\tanh \tau
$$

and

$$
d \mathfrak{s}_{S^{5}, \text { induced }}^{2}=\frac{1}{\cosh ^{2} \tau}\left(d \tau^{2}+d s^{2}\right) .
$$

Changing the world-sheet coordinates from $(\tau, s)$ to $(z, s)$ in (4.16), (4.18) we get back to (4.11). Computation of the area in the conformal gauge, of course, gives the same result:

$$
A(\text { circle })=2 \pi \int_{\varepsilon}^{\infty} d \tau\left(\frac{1}{\sinh ^{2} \tau}+\frac{1}{\cosh ^{2} \tau}\right)=\left.2 \pi(\tanh \tau-\operatorname{coth} \tau)\right|_{\varepsilon} ^{\infty}=\frac{2 \pi}{\varepsilon} \rightarrow 0 .
$$


The above solution for the minimal surface depends on an arbitrary point on $S^{3}$, denoted by $\mathbf{n}$ in (4.8). Different $\mathbf{n}$ 's correspond to different minimal surfaces, so the solution indeed depends on three arbitrary parameters, exactly as predicted by supersymmetry! Why minimal surfaces that sit at one point in $S^{5}$ do not have this degeneracy? The reason is that $S^{3}$ shrinks to zero size at $\psi=0 .(\psi=0, \mathbf{n})$ correspond to one point in $S^{5}$ for all $\mathbf{n}$. As soon as $\Psi$ is identically equal to zero, the degeneracy does not arise. It is easy to see that the three-parametric degeneracy is not specific to the circular loop. Indeed, any planar contour projects onto an equatorial circle in $S^{5}$ under the map defined in sec. 2. For symmetry reasons, the minimal surface will not extend into the orthogonal $S^{3}$, coordinates on which can be regarded as moduli parameterizing the minimal surface.

It is natural to assume that $\left\langle W_{s}(C)\right\rangle=1$ for any planar loop $C$. This conjecture relies on the nullification of the minimal area in two cases, for the anti-parallel lines [26] and for the circular loop considered above. It would clearly be desirable to have a general proof that the minimal area is zero for any planar contour. On the other hand, cancellation of zero modes simply follows from the ' $3+1$ ' decomposition of the metric in eq. (4.7) and applies to any $1 / 4$ BPS Wilson loop.

What about less supersymmetric loops? A $d$-dimensional contour in $\mathbb{R}^{4}$ maps onto a curve in the equatorial $S^{d-1}$ in $S^{5}$. It is then convenient to decompose the $S^{5}$ metric in the ' $(d-1)+(5-d)$ ' way:

$$
d \Omega_{S^{5}}^{2}=d \psi^{2}+\cos ^{2} \psi d \Omega_{S^{d-1}}^{2}+\sin ^{2} \psi d \Omega_{S^{5-d}}^{2}
$$

The minimal surface will sit at one point in $S^{5-d}$. So, the solution for the minimal surface will contain $N_{z . m .}=(5-d)$ moduli. Consequently, the expectation value for a $d$-dimensional supersymmetric Wilson loop is proportional to ${ }^{\ddagger}$

$$
\left\langle W_{s}\left(C_{d}\right)\right\rangle \propto\left(g^{2} N\right)^{(2-d) / 4} \exp \left(-\frac{\sqrt{g^{2} N}}{2 \pi} A(C)\right) .
$$

If $d>2$, the expectation value is a non-trivial function of the 't Hooft coupling, whatever the minimal area is. There are no reasons to expect that the minimal area for non-planar contours is zero, since non-renormalization does not work in this case anyway.

\section{Discussion}

The supersymmetric Wilson loops can be constructed in $\mathcal{N}=2$ SYM theory as well. The details are given in Appendix. These operators can be useful in the study of supergravity duals of $\mathcal{N}=2$ gauge theories constructed in [39]-[42].

It is likely that planar supersymmetric Wilson loops in $\mathcal{N}=4 \mathrm{SYM}$, which preserve $1 / 4$ of the supersymmetry, are not renormalized by quantum corrections. At strong coupling, non-renormalization of planar Wilson loops implies cancellation of the minimal area in $A d S_{5} \times S^{5}$ for rather wide class of boundary conditions. This is a very

\footnotetext{
$\ddagger$ This formula is not applicable at $d=1$ (the straight line), because of boundary conditions at spatial infinity that should be imposed in this case. These extra boundary conditions modify zero mode counting. Properly defined partition function for the straight Wilson line should be equal to one.
} 
non-trivial statement about minimal surfaces in $A d S_{5} \times S^{5}$. I did not prove it, but checked for the circular and the rectangular loops.

Wilson loops with lesser degree of supersymmetry do renormalize. A non-trivial dependence on 't Hooft coupling follows from zero mode counting in the string partition function. The dependence of non-planar loops on 't Hooft coupling and the couplingconstant independence of planar loops has an interesting consequence. Consider a slightly non-planar Wilson loop; let $\delta$ be a parameter of non-planarity. The strongcoupling asymptotics of the Wilson loop vev then is a discontinuous function of $\delta$. This can be seen from the structure of zero-mode contribution (a pre-factor in (4.22)) to the string partition function. The number of zero modes changes as $\delta$ turns to zero, so the expectation values for $\delta=0$ and for infinitesimally small but non-zero $\delta$ differ by a finite amount. Such a discontinuity is absent in any order of perturbation theory, as any Feynman diagram is an analytic function of $\delta$. Similar strong-coupling 'phase transitions' occur in Wilson loop correlators [43]-[47], they arise due to the string breaking.

\section{Acknowledgements}

I am grateful to Anton Alekseev for hospitality at the University of Geneva, where this work was completed. The work was supported by STINT grant IG 2001-062 and by Royal Swedish Academy of Sciences and, in part, by RFBR grant 02-02-17260 and grant 00-15-96557 for the promotion of scientific schools.

\section{Appendix A Supersymmetric Wilson loops in $\mathcal{N}=2$ SYM theory}

The field content of $\mathcal{N}=2 \mathrm{SYM}$ theory consists of the gauge fields $A_{\mu}$, two Majorana fermions $\lambda_{\alpha}^{A}, A=1,2$, and a complex scalar $\Phi$. All fields are in the adjoint representation of the gauge group, $S U(N)$. The supersymmetry transformations of the bosonic fields are:

$$
\begin{aligned}
\delta A_{\mu} & =-i \bar{\lambda}_{A} \bar{\sigma}_{\mu} \epsilon^{A}+i \bar{\epsilon}_{A} \bar{\sigma}_{\mu} \lambda^{A} \\
\delta \Phi & =\sqrt{2} \varepsilon_{A B} \epsilon^{A} \lambda^{B} \\
\delta \Phi^{\dagger} & =-\sqrt{2} \varepsilon^{A B} \bar{\epsilon}_{A} \bar{\lambda}_{B},
\end{aligned}
$$

where $\epsilon^{A}$ are two Majorana spinors, and I follow the conventions of [48]. In particular, I switch to the Minkowski metric with signature $(-+++)$.

The supersymmetric Wilson loop is defined as

$$
W_{s}(C, \zeta)=\frac{1}{N} \operatorname{tr} \mathrm{P} \exp \int d s\left(i A_{\mu} \dot{x}^{\mu}+\Phi \zeta^{*}|\dot{x}|+\Phi^{*} \zeta|\dot{x}|\right),
$$

where $\zeta \equiv \zeta(s)$ is an arbitrary complex-valued function of the parameter on the contour, which satisfies $|\zeta|=1 / \sqrt{2}$. The modulus of the tangent vector, $|\dot{x}|$, stands for $\sqrt{\dot{x}^{2}}$ and is imaginary for time-like contours. Therefore, time-like Wilson loops are associated with unitary matrices, as usual, and it is only space-like loops that contain a Hermitian piece in the exponent. Light-like loops do not couple to scalars at all. 
The requirement that the supersymmetry variation of the Wilson loop turns to zero is equivalent to the following equations:

$$
\begin{aligned}
\bar{\sigma}^{\mu} \dot{x}_{\mu} \epsilon^{A} & =-\sqrt{2}|\dot{x}| \zeta \varepsilon^{A B} \bar{\epsilon}_{B}, \\
\bar{\epsilon}_{A} \bar{\sigma}^{\mu} \dot{x}_{\mu} & =\sqrt{2}|\dot{x}| \zeta^{*} \epsilon^{B} \varepsilon_{B A} .
\end{aligned}
$$

These equations are Hermitian conjugates of one another. The first equation can be used to express $\bar{\epsilon}_{A}$ in terms of $\epsilon^{A}$ :

$$
\bar{\epsilon}_{A}=-\frac{1}{\sqrt{2} \zeta|\dot{x}|} \varepsilon_{A B} \bar{\sigma}^{\mu} \dot{x}_{\mu} \epsilon^{B}
$$

Substituting this into the second equation we get

$$
\bar{\sigma}^{\mu \dot{\alpha} \alpha} \varepsilon_{\dot{\alpha} \dot{\beta}} \bar{\sigma}^{\nu \dot{\beta} \beta} \dot{x}_{\mu} \dot{x}_{\nu} \varepsilon_{A B} \epsilon_{\alpha}^{B}=-2|\zeta|^{2} \dot{x}^{2} \varepsilon_{A B} \epsilon^{B \beta},
$$

which can be shown to be an identity, provided $2|\zeta|^{2}=1$.

The four constraints (A.4) fix half of the parameters of the supersymmetry transformation. Again, generic Wilson loop preserves only local supersymmetry, since the right hand side of (A.4) in general depends on a position on the curve. An obvious exception is the straight line with constant $\zeta$, which preserves $1 / 2$ of global $\mathcal{N}=2$ supersymmetry. It would be interesting to understand if there are other BPS Wilson loops, which preserve smaller part of the supersymmetry.

\section{References}

[1] J. Maldacena, "The large N limit of super-conformal field theories and supergravity," Adv. Theor. Math. Phys. 2, 231 (1998) [Int. J. Theor. Phys. 38, 1113 (1998)] [hep-th/9711200].

[2] S. S. Gubser, I. R. Klebanov and A. M. Polyakov, "Gauge theory correlators from non-critical string theory," Phys. Lett. B 428 (1998) 105 [arXiv:hep-th/9802109].

[3] E. Witten, "Anti-de Sitter space and holography," Adv. Theor. Math. Phys. 2, 253 (1998) [hep-th/9802150].

[4] O. Aharony, S. S. Gubser, J. Maldacena, H. Ooguri and Y. Oz, "Large N field theories, string theory and gravity," Phys. Rept. 323 (2000) 183 [arXiv:hepth/9905111].

[5] G. 't Hooft, "A Planar Diagram Theory For Strong Interactions," Nucl. Phys. B 72, 461 (1974).

[6] S.S. Gubser and I.R. Klebanov, "Absorption by branes and Schwinger terms in the world volume theory," Phys. Lett. B413, 41 (1997) [arXiv:hep-th/9708005].

[7] D. Anselmi, D.Z. Freedman, M.T. Grisaru and A.A. Johansen, "Nonperturbative formulas for central functions of supersymmetric gauge theories," Nucl. Phys. B526, 543 (1998) [arXiv:hep-th/9708042].

[8] D.Z. Freedman, S..D. Mathur, A. Matusis and L. Rastelli, "Correlation functions in the CFT(d) / AdS $(d+1)$ correspondence," Nucl. Phys. B546, 96 (1999) [arXiv:hep-th/9804058]. 
[9] S. Lee, S. Minwalla, M. Rangamani and N. Seiberg, "Three point functions of chiral operators in $\mathrm{D}=4, \mathrm{~N}=4 \mathrm{SYM}$ at large N," Adv. Theor. Math. Phys. 2, 697 (1998) [arXiv:hep-th/9806074].

[10] E. D'Hoker, D.Z. Freedman and W. Skiba, "Field theory tests for correlators in the AdS / CFT correspondence," Phys. Rev. D59, 045008 (1999) [arXiv:hepth/9807098].

[11] K. A. Intriligator, "Bonus symmetries of $\mathrm{N}=4$ super-Yang-Mills correlation functions via AdS duality," Nucl. Phys. B 551 (1999) 575 [arXiv:hep-th/9811047].

[12] F. Gonzalez-Rey, B. Kulik and I. Y. Park, "Non-renormalization of two point and three point correlators of $\mathrm{N}=4 \mathrm{SYM}$ in $\mathrm{N}=1$ superspace," Phys. Lett. B 455 (1999) 164 [arXiv:hep-th/9903094].

[13] K. A. Intriligator and W. Skiba, "Bonus symmetry and the operator product expansion of N = 4 super-Yang-Mills," Nucl. Phys. B 559 (1999) 165 [arXiv:hepth/9905020].

[14] B. Eden, P. S. Howe and P. C. West, "Nilpotent invariants in N = 4 SYM," Phys. Lett. B 463 (1999) 19 [arXiv:hep-th/9905085].

[15] A. Petkou and K. Skenderis, "A non-renormalization theorem for conformal anomalies," Nucl. Phys. B 561 (1999) 100 [arXiv:hep-th/9906030].

[16] H. Liu and A. A. Tseytlin, "Dilaton-fixed scalar correlators and AdS(5) x S(5) SYM correspondence," JHEP 9910 (1999) 003 [arXiv:hep-th/9906151].

[17] S. Penati, A. Santambrogio and D. Zanon, "Two-point functions of chiral operators in N = 4 SYM at order $g^{4}$," JHEP 9912 (1999) 006 [arXiv:hep-th/9910197]; "More on correlators and contact terms in $\mathrm{N}=4$ SYM at order $g^{4}$," Nucl. Phys. B 593 (2001) 651 [arXiv:hep-th/0005223].

[18] E. D'Hoker and D. Z. Freedman, "Supersymmetric gauge theories and the AdS/CFT correspondence," arXiv:hep-th/0201253.

[19] J. K. Erickson, G. W. Semenoff and K. Zarembo, "Wilson loops in N = 4 supersymmetric Yang-Mills theory," Nucl. Phys. B 582, 155 (2000) [arXiv:hepth/0003055].

[20] N. Drukker, D. J. Gross and A. Tseytlin, "Green-Schwarz string in AdS(5) x S(5): Semiclassical partition function," JHEP0004, 021 (2000) [arXiv:hep-th/0001204].

[21] N. Drukker and D. J. Gross, "An exact prediction of $N=4$ SUSYM theory for string theory," J. Math. Phys. 42 (2001) 2896 [arXiv:hep-th/0010274].

[22] G. W. Semenoff and K. Zarembo, "Wilson loops in SYM theory: From weak to strong coupling," Nucl. Phys. Proc. Suppl. 108 (2002) 106 [short version of arXiv:hep-th/0202156].

[23] G. W. Semenoff and K. Zarembo, "More exact predictions of SUSYM for string theory," Nucl. Phys. B 616 (2001) 34 [arXiv:hep-th/0106015].

[24] N. Drukker, D. J. Gross and H. Ooguri, "Wilson loops and minimal surfaces," Phys. Rev. D 60, 125006 (1999) [arXiv:hep-th/9904191].

[25] M. Bianchi, M. B. Green and S. Kovacs, "Instanton corrections to circular Wilson loops in N = 4 supersymmetric Yang-Mills," JHEP 0204 (2002) 040 [arXiv:hepth/0202003]. 
[26] J. Maldacena, "Wilson loops in large N field theories," Phys. Rev. Lett. 80, 4859 (1998) [arXiv:hep-th/9803002].

[27] Y. M. Makeenko, "Polygon Discretization Of The Loop Space Equation," Phys. Lett. B 212 (1988) 221.

[28] J. Plefka and M. Staudacher, "Two loops to two loops in $\mathrm{N}=4$ supersymmetric Yang-Mills theory," JHEP 0109, 031 (2001) [arXiv:hep-th/0108182].

[29] G. Arutyunov, J. Plefka and M. Staudacher, "Limiting geometries of two circular Maldacena-Wilson loop operators," JHEP 0112 (2001) 014 [arXiv:hepth/0111290].

[30] S. J. Rey and J. Yee, "Macroscopic strings as heavy quarks in large N gauge theory and anti-de Sitter supergravity," Eur. Phys. J. C 22 (2001) 379 [arXiv:hepth/9803001].

[31] R. R. Metsaev and A. A. Tseytlin, "Type IIB superstring action in AdS(5) x S(5) background," Nucl. Phys. B 533 (1998) 109 [arXiv:hep-th/9805028].

[32] R. Kallosh, J. Rahmfeld and A. Rajaraman, "Near horizon superspace," JHEP 9809 (1998) 002 [arXiv:hep-th/9805217].

[33] I. Pesando, "A kappa gauge fixed type IIB superstring action on $\operatorname{AdS}(5)$ x S(5)," JHEP 9811 (1998) 002 [arXiv:hep-th/9808020].

[34] R. Kallosh and J. Rahmfeld, "The GS string action on AdS(5) x S(5)," Phys. Lett. B 443 (1998) 143 [arXiv:hep-th/9808038].

[35] R. Kallosh and A. A. Tseytlin, "Simplifying superstring action on AdS(5) x S(5)," JHEP 9810 (1998) 016 [arXiv:hep-th/9808088].

[36] S. Frolov and A. A. Tseytlin, "Semiclassical quantization of rotating superstring in $A d S_{5} \times S^{5}$," JHEP 0206 (2002) 007 [arXiv:hep-th/0204226].

[37] O. Alvarez, "Theory Of Strings With Boundaries: Fluctuations, Topology, And Quantum Geometry," Nucl. Phys. B 216 (1983) 125.

[38] D. Berenstein, R. Corrado, W. Fischler and J. Maldacena, "The operator product expansion for Wilson loops and surfaces in the large N limit," Phys. Rev. D 59, 105023 (1999) [arXiv:hep-th/9809188].

[39] M. Bertolini, P. Di Vecchia, M. Frau, A. Lerda, R. Marotta and I. Pesando, "Fractional D-branes and their gauge duals," JHEP 0102 (2001) 014 [arXiv:hepth/0011077].

[40] J. Polchinski, "N=2 gauge-gravity duals," Int. J. Mod. Phys. A 16 (2001) 707 [arXiv:hep-th/0011193].

[41] M. Bertolini, P. Di Vecchia, M. Frau, A. Lerda and R. Marotta, "N = 2 gauge theories on systems of fractional D3/D7 branes," Nucl. Phys. B 621 (2002) 157 [arXiv:hep-th/0107057].

[42] M. Bertolini, P. Di Vecchia and R. Marotta, " $\mathrm{N}=2$ four-dimensional gauge theories from fractional branes," arXiv:hep-th/0112195.

[43] D. J. Gross and H. Ooguri, "Aspects of large N gauge theory dynamics as seen by string theory," Phys. Rev. D 58 (1998) 106002 [arXiv:hep-th/9805129]. 
[44] K. Zarembo, "Wilson loop correlator in the AdS/CFT correspondence," Phys. Lett. B 459 (1999) 527 [arXiv:hep-th/9904149].

[45] P. Olesen and K. Zarembo, "Phase transition in Wilson loop correlator from AdS/CFT correspondence," arXiv:hep-th/0009210.

[46] H. Kim, D. K. Park, S. Tamarian and H. J. Muller-Kirsten, "Gross-Ooguri phase transition at zero and finite temperature: Two circular Wilson loop case," JHEP 0103 (2001) 003 [arXiv:hep-th/0101235].

[47] K. Zarembo, "String breaking from ladder diagrams in SYM theory," JHEP 0103, 042 (2001) [hep-th/0103058].

[48] J.Wess and J. Bagger, Supersymmetry and Supergravity (Princeton University Press, 1982). 